\title{
Arguments en faveur d'une norme canadienne pour l'intégration des enjeux 2SLGBTQIA+ dans les études de médecine
}

\author{
Miranda Schreiber BA, Tehmina Ahmad MD, Michael Scott MD, Kevin Imrie MD MBA, Saleem Razack MD
}

Citation : CMAJ 2021 April 19;193:E562-5. doi : 10.1503/cmaj.202642-f

Voir la version anglaise de l'article ici : www.cmaj.ca/lookup/doi/10.1503/cmaj.202642

A

u Canada, les personnes bispirituelles, lesbiennes, gaies, bisexuelles, transgenres, queers, intersexes et asexuelles (2SLGBTQIA+) ont une santé plus précaire que les personnes hétérosexuelles cisgenres. L'oppression de longue date de la communauté 2SLGBTQIA+ - oppression à laquelle le système de santé n'est pas étranger - favorise ces disparités. Au XIXe siècle, dans la médecine occidentale, l'homosexualité était considérée comme une maladie. Jusqu'au début des années 1970, elle était classée parmi les pathologies dans le Manuel diagnostique et statistique des troubles mentaux (DSM), où la dysphorie de genre est d'ailleurs, aujourd'hui encore, définie comme un trouble de santé mentale. De nos jours, les expériences négatives auprès de professionnels de la santé poussent les personnes 2SLGBTQIA+ à s'abstenir d'aller chercher des soins, et bien des médecins disent se sentir mal préparés pour soigner les membres de cette communauté. Au Canada et aux États-Unis, les éducateurs du secteur médical ont reconnu le besoin de préparer les étudiants à prodiguer des soins éclairés et empreints de compassion aux patients $2 \mathrm{SLGBTQIA+}{ }^{1}$, mais la formation sur la santé de ce groupe reste limitée et inégale. C'est pourquoi nous nous penchons sur les promesses et les difficultés liées à l'intégration de contenu 2SLGBTQIA+ dans la formation offerte par les facultés de médecine canadiennes. Nous demandons l'instauration d'une norme nationale en matière de formation et d'objectifs de compétence liés aux enjeux 2SLGBTQIA+ pour les médecins au Canada.

\section{Quels facteurs influencent la santé des personnes 2SLGBTQIA+ au Canada?}

Selon le rapport de 2019 du Comité permanent de la santé de la Chambre des communes sur la santé des communautés LGBTQIA2, les membres de ces communautés ont des résultats de santé inférieurs à ceux de leurs homologues cisgenres hétérosexuels ${ }^{2}$ : ils sont notamment touchés de façon disproportionnée par le cancer, la fatigue chronique et les cardiopathies ${ }^{2}$. Le rapport montre que les personnes 2SLGBTQIA+ sont

\section{POINTS CLÉS}

- Au Canada, les personnes bispirituelles, lesbiennes, gaies, bisexuelles, transgenres, queers, intersexes et asexuelles (2SLGBTQIA+) ont des résultats de santé disproportionnellement inférieurs à ceux du reste de la population.

- La combinaison de systèmes d'oppression, comme la suprématie blanche et le colonialisme, génère des résultats de santé inférieurs pour les personnes 2SLGBTQIA+ noires et autochtones.

- Les fournisseurs de soins de santé ne sont pas adéquatement formés sur la santé des personnes 2SLGBTQIA+, ce qui entraîne des expériences de soins de qualité moindre pour ces patients.

- L'intégration de contenu 2SLGBTQIA+ dans les programmes d'études de médecine n'est pas sans difficulté, mais il s'agit d'un moyen éprouvé et efficace d'améliorer les compétences.

- La mise en place d'une norme nationale exigeant l'inclusion des enjeux 2SLGBTQIA+ dans les études de médecine de premier cycle et des cycles supérieurs améliorera les soins offerts à cette communauté.

moins susceptibles d'avoir un médecin de famille et plus susceptibles d'être atteintes d'une maladie chronique, de problèmes de santé mentale, et de toxicomanie ${ }^{2,3}$.

Ces disparités sont dues à l'oppression structurelle passée et présente des personnes 2SLGBTQIA+. La pathologisation de I'homosexualité au XIXe siècle a mené les médecins occidentaux à soumettre les personnes 2SLGBTQIA+ à des lobotomies, à des électrochocs et à la castration chimique jusqu'aux années $1970^{4}$. Dans le DSM, l'homosexualité était répertoriée parmi les troubles mentaux jusqu'en 1973, et dans l'édition actuelle (publiée en 2013) la " dysphorie de genre » est classée comme un trouble mental, ce qui a pour effet de pathologiser l'état des personnes transgenres ${ }^{4,5}$. La crise du sida, à son apogée dans les années 1980, a été traitée avec négligence par les gouvernements canadien et américain. Le gouvernement Reagan rechignait à financer la recherche sur la maladie, et il a fallu attendre 1985 pour qu'il reconnaisse l'épidémie ${ }^{6}$. Élu 
premier ministre du Canada en 1984, Brian Mulroney n'a pas admis l'existence du sida avant 1989, ce qui a forcé les groupes 2SLGBTQIA+ et les chercheurs canadiens à tenter de fournir des traitements expérimentaux aux malades sans financement public.

En 2021, les injustices structurelles perpétuent le retard en santé de la communauté 2SLGBTQIA+. Une part disproportionnée des membres de cette communauté vit en situation d'itinérance : les jeunes 2SLGBTQIA+, qui comptent pour $5 \%$ de cette tranche d'âge, représentent $40 \%$ des jeunes sansabri de Toronto ${ }^{9,10}$. Comme l'accès au logement est l'un des principaux déterminants de la santé, la crise du logement actuelle pénalise de façon démesurée les personnes 2 SLGBTQIA+9,10. La surreprésentation de ces personnes - surtout si elles sont noires ou autochtones - dans les emplois mal rémunérés et l'insuffisance du soutien public aux démunis contribuent également à la santé sous-optimale de la communauté 2 SLGBTQIA ${ }^{11}$.

Le système médical lui-même perpétue aussi les disparités. Aux États-Unis, des sondages nationaux et étatiques ont révélé que les expériences négatives en milieu médical poussent les personnes 2SLGBTQIA+ à s'abstenir d'aller chercher des soins, et selon une étude menée auprès de groupes de discussion en 2011, les patients 2SLGBTQIA+ se sentent obligés de renseigner eux-mêmes les professionnels qui leur fournissent des soins sur leur réalité ${ }^{12,13}$. Un sondage effectué en 2017 auprès de personnes transgenres en Ontario a révélé que $43 \%$ d'entre elles avaient eu un besoin de santé non comblé au cours de l'année précédente ${ }^{14}$. Un autre sondage de 2017, mené auprès de 45 professionnels de la santé en exercice, a fait ressortir des lacunes systématiques dans les connaissances au sujet de la santé de la communauté 2SLGBTQIA+; une analyse documentaire sur les facteurs de risque connus liés à la santé des personnes 2SLGBTQIA+ a indiqué que les professionnels de la santé négligeaient ces variables dans leurs entretiens avec les patients ${ }^{15,16}$. L'incapacité à fournir suffisamment d'évaluations fondées sur des données probantes a par ailleurs mené des professionnels de la santé à donner des conseils inexacts qui ont eu pour effet d'accroître le risque de cancer du col de l'utérus de personnes 2SLGBTQIA $+{ }^{17}$.

Une analyse intersectionnelle ${ }^{18}$ montre qu'au Canada, la combinaison de multiples systèmes d'oppression engendre des difficultés particulières pour les personnes 2SLGBTQIA+ désavantagées par d'autres systèmes. Par exemple, les personnes bispirituelles voient leur accès aux soins entravé par le colonialisme, le racisme et l'insensibilité culturelle, qui s'ajoutent à l'homophobie et à l'hétérosexisme ${ }^{19-21}$. En outre, des études qualitatives menées dans la population canadienne indiquent que les hommes noirs homosexuels rencontrent aussi des difficultés particulières et non négligeables dans l'accès aux soins ${ }^{22}$, et que les personnes 2SLGBTQIA+ qui immigrent d'Afrique et des Caraïbes souffrent de façon disproportionnée de traumatismes et de troubles de santé mentale $e^{23}$. Comme le conclut le rapport du Comité permanent de la santé de la Chambre des communes, "chaque sous-groupe des communautés LGBTQIA2 présente des vulnérabilités spécifiques en matière de santé et [...] une analyse intersectionnelle est requise $»^{2}$.

\section{Comment les enjeux 2SLGBTQIA+ sont-ils actuellement enseignés dans les facultés de médecine américaines et canadiennes?}

Au Canada et aux États-Unis, la formation sur la santé des personnes 2SLGBTQIA+ est actuellement limitée et inégale ${ }^{24}$. Les facultés n'ont pas réussi à créer et à officialiser du contenu à ce sujet, et elles perpétuent les préjugés courants et la mésinformation sur la communauté 2SLGBTQIA+. Un sondage mené dans toutes les facultés de médecine américaines en 1991 faisait état d'une moyenne de 3 heures 26 minutes d'étude consacrée à la santé des patients homosexuels ${ }^{25}$. Vingt ans plus tard, un sondage des facultés de médecine nord-américaines a révélé que le nombre d'heures médian accordé à l'enseignement de contenu sur les réalités LGBT, sur 4 ans, était de seulement 5 heures, que les étudiants manquaient d'exposition clinique et que moins de $35 \%$ de ces facultés traitaient de la santé des personnes transgenres ${ }^{24}$. Selon un sondage national de 2016, si $95 \%$ des étudiants en médecine canadiens reconnaissaient l'importance des soins adaptés aux personnes transgenres, moins de $10 \%$ se sentaient assez outillés pour prodiguer de tels soins ${ }^{26}$.

Les lacunes relatives à la santé de la communauté 2SLGBTQIA+ dans les études de médecine de premier cycle ont des effets en aval sur les attitudes et les compétences des médecins. En 2019, une évaluation multicentrique du degré de préparation des médecins résidents à traiter les patients 2SLGBTQIA+ a été effectuée en ligne et a montré que les étudiants de tous les niveaux manquaient de préparation en ce qui a trait à la terminologie, à la connaissance des soins préventifs, aux soins psychologiques et à la santé sexuelle ${ }^{27}$. Selon cette évaluation, les étudiants ne savaient pas qu'il existait des disparités en santé propres à cette communauté $^{27}$. Les programmes de médecine d'urgence n'offrent en moyenne que 45 minutes d'enseignement consacré spécifiquement aux patients LGBT ${ }^{28}$. Enfin, $80 \%$ des endocrinologues en exercice n'avaient pas reçu de formation sur les soins aux personnes transgenres, même si $80 \%$ des répondants disaient avoir traité des personnes transgenres ${ }^{29}$.

En 2015, l'Association des écoles de médecine des ÉtatsUnis a déclaré que l'absence de formation médicale sur la santé des personnes 2SLGBTQIA+, conjuguée au climat conservateur des établissements d'enseignement, produisait un curriculum hétéronormatif et cisnormatif caché sousentendant que la santé des personnes 2SLGBTQIA+ n'avait pas d'importance ${ }^{1}$. Dans un sondage mené en 2017 auprès d'étudiants en médecine de l'Université d'Ottawa, $41 \%$ des répondants ont dit avoir été témoins de comportements intolérants à l'égard des personnes LGBT de la part de leurs pairs ou de professeurs ${ }^{30}$. C'est sans compter que les questions des examens de médecine perpétuent les idées reçues en associant continuellement les scénarios de 
patients 2SLGBTQIA+ avec le VIH et les infections transmissibles sexuellement ${ }^{31}$.

L'Association des écoles de médecine des États-Unis a indiqué que les institutions médicales devaient « préparer les étudiants à répondre avec efficacité, compassion et professionnalisme aux besoins de tous les types de patients [...], quelle que soit leur orientation sexuelle ou leur identité de genre ${ }^{32}$. » L'Association a en outre désigné l'« absence de contenu LGBT » dans les examens nationaux des organismes d'agrément comme une lacune importante des normes d'éducation ${ }^{32}$.

\section{Quels sont les obstacles à la mise sur pied d'un programme d'études offrant une formation médicale adéquate sur les enjeux 2SLGBTQIA+?}

L'absence de données médicales portant précisément sur les personnes 2SLGBTQIA+ complique l'élaboration de programmes d'études sur la réalité de ces personnes. En mai 2021, le Recensement du Canada recueillera pour la première fois des données multidimensionnelles sur l'appartenance à la communauté 2SLGBTQIA+; le nombre exact de personnes 2SLGBTQIA+ au pays n'est pas connu avec certitude ${ }^{2}$. De plus, on en sait peu sur les méthodes qui servent le mieux les étudiants et les patients 2SLGBTQIA+, car peu d'approches pédagogiques ont été évaluées ${ }^{33}$. La faible distribution de ressources complètes sur les enjeux 2SLGBTQIA+ aux étudiants et aux médecins résidents rend d'autant plus difficiles la collaboration et la recherche, surtout vu la rareté des occasions pour les experts de la santé 2SLGBTQIA+ de travailler avec les futurs médecins.

\section{L'amélioration de la formation médicale aurait-elle une incidence positive sur la santé des personnes 2SLGBTQIA+ au Canada?}

Comme l'ont observé Sawning et ses collaborateurs, "le manque de formation des fournisseurs de soins n'est qu'un des facteurs » expliquant les disparités; la modification des programmes d'études ne réglera pas tous les problèmes ${ }^{34,35}$. La mise sur pied de programmes traitant des enjeux 2SLGBTQIA+ a ses limites, puisqu'elle ne fait rien contre les structures d'oppression à l'origine des disparités en santé pour la communauté 2SLGBTQIA+, comme la suprématie blanche, le colonialisme et la crise du logement ${ }^{36}$.

Malgré tout, les experts, les membres de la communauté, les chercheurs et les apprenants recommandent d'intégrer du contenu sur les réalités 2SLGBTQIA+ aux programmes d'études de médecine pour réduire la proportion de résultats sousoptimaux en matière de santé. Le Comité de la Chambre des communes, dans ses recommandations de mesures à prendre pour réduire les écarts en santé, propose l'amélioration de la formation de tous les fournisseurs de soins au sujet des

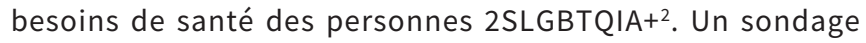
mené auprès d'étudiants en médecine canadiens a également indiqué que des changements fondamentaux aux programmes d'études pourraient faire mieux connaître les soins de santé adaptés aux personnes transgenres ${ }^{26}$. Enfin, il est de plus en plus recommandé dans les études de former les professionnels de la santé en suivant des normes préétablies concernant les besoins concomitants de certaines communautés, par exemple les réfugiés et les migrants 2SLGBTQIA+, ou encore les femmes séropositives ${ }^{23,35}$.

Il a été démontré que l'intégration de contenu sur la santé de la communauté 2SLGBTQIA+ dans les programmes d'études de médecine améliore d'une part les connaissances des médecins en ce qui a trait à ces groupes, et d'autre part l'expérience de soins des personnes 2SLGBTQIA+. Des études menées sur des ateliers traitant des enjeux 2SLGBTQIA+ à l'École de médecine du Nord de l'Ontario et à l'Université de Louisiane ont permis d'établir que ce type d'intervention était efficace et formateur, et qu'il révélait des lacunes dans les connaissances des apprenants; les chercheurs ont ainsi conclu que la formation médicale pouvait contribuer à l'élimination des disparités en santé ${ }^{33,35}$. Même l'ajout de quelques heures de formation sur les enjeux 2SLGBTQIA+ suffisait à augmenter la confiance des apprenants au Canada et aux États-Unis quant à leur capacité à traiter des patients appartenant à cette communauté ${ }^{33,37}$. On observe une corrélation entre l'augmentation de la place accordée au contenu 2SLGBTQIA+ dans les études de médecine et la qualité des questions sur les antécédents sexuels, la rigueur des conseils de prévention et la proportion de signes de préjugés au moment de l'admission de nouveaux patients ${ }^{33}$. L'intégration de contenu 2SLGBTQIA+ dans les programmes d'études est donc une solution incomplète, mais non sans importance pour les personnes 2SLGBTQIA+.

\section{Comment intégrer le contenu 2SLGBTQIA+ aux programmes d'études de médecine de premier cycle et des cycles supérieurs?}

Au moment de la rédaction du présent article, l'Association des facultés de médecine du Canada, le Collège royal des médecins et chirurgiens du Canada et le Collège des médecins de famille du Canada n'avaient aucune cible d'évaluation explicite exigeant des médecins résidents et des étudiants en médecine qu'ils démontrent des connaissances ou des capacités de gestion en ce qui a trait précisément au traitement des patients 2SLGBTQIA+. Bien que les facultés de médecine du Canada soient membres de l'Association des écoles de médecine des États-Unis et aient accès à ses ressources, aucun organisme de réglementation canadien n'oblige les étudiants et les professionnels du domaine de la santé à démontrer leur compétence à prodiguer des soins aux personnes 2SLGBTQIA+. L'Association des écoles de médecine des États-Unis a établi une liste de compétences relatives à la santé des personnes LGBTQ, mais nous soutenons que les guides conçus pour les besoins et le système de santé des États-Unis ne sont pas adaptés à la situation des personnes 2SLGBTQIA+ au Canada, en particulier celles qui sont noires ou autochtones. 
L'évolution des approches pédagogiques observée ces 10 dernières années est favorable aux changements requis dans les programmes de médecine. La formation axée sur les compétences permet de commencer à intégrer les besoins de santé de la communauté 2SLGBTQIA+ dans les évaluations et les soins cliniques ${ }^{34,38,39}$. Les changements pourraient se faire en 3 phases.

D'abord, pour améliorer la connaissance des soins de santé adaptés aux patients 2SLGBTQIA+ chez les professionnels du milieu, il y a lieu de faire une plus grande place au contenu 2SLGBTQIA+ dans les programmes d'études au moyen de l'apprentissage par mises en situation, d'entrevues normalisées avec des patients et de conférences didactiques longitudinales tout au long de la formation de premier cycle et des cycles supérieurs ${ }^{1}$. L'intégration de ces thèmes doit se faire en collaboration avec des organisations et des patients 2SLGBTQIA+ et doit tenir compte des obstacles systémiques à la santé pour les personnes racisées, surtout celles qui sont noires ou autochtones ${ }^{23,39}$. Il faut établir des liens entre les écarts en santé et les structures d'oppression qui les produisent ${ }^{40}$. Les conférences sur les enjeux propres aux patients 2SLGBTQIA+ pourraient traiter de terminologie, d'histoire, de méthodes d'examen physique, d'évaluations pour l'hormonothérapie, de pratiques sexuelles sécuritaires, de fertilité, de dépistage du cancer, de santé mentale et de problèmes de toxicomanie. Il ne suffit pas d'ajouter ce contenu aux programmes : il importe de montrer que la santé des personnes 2SLGBTQIA+ compte dans toutes les disciplines de la médecine ${ }^{41}$. En outre, en fournissant des ressources qui permettraient aux étudiants, au personnel, aux patients et aux médecins appartenant à la communauté 2SLGBTQIA+ de collaborer à l'élaboration des programmes d'études ou à des projets de communication ou de recherche, il serait possible de véhiculer rapidement les connaissances sur la santé des patients 2SLGBTQIA+.

Ensuite, afin d'améliorer la qualité des consultations pour les personnes 2SLGBTQIA+, les programmes pourraient apprendre aux futurs médecins à interagir avec les patients 2SLGBTQIA+ dans des entrevues normalisées avec des patients et dans leurs stages cliniques. Les mises en situation cliniques devraient tenir compte des traumatismes, montrer à éviter les suppositions sur l'identité et traiter de racisme dans le milieu médical, de santé mentale, de traumatismes liés aux soins, de santé sexuelle et de toxicomanie. Les mises en situation montreraient les besoins de différents groupes d'âge et comporteraient une analyse intersectionnelle; les scénarios enseigneraient aussi la façon d'orienter les patients vers les ressources communautaires et le soutien dont ils ont besoin ${ }^{34,39-41}$. Les facultés de médecine et les milieux d'enseignement doivent être des environnements propices à l'épanouissement des étudiants et du personnel 2SLGBTQIA+, pour que l'apprentissage des soins respectueux soit autant implicite qu'explicite ${ }^{1}$.

Enfin, pour mener à bien des changements structurels et plus largement pour cibler les causes du retard en santé de la communauté 2SLGBTQIA+, les établissements de santé du Canada doivent s'interroger sur leur rôle, en tant qu'institu- tions d'élite, dans la perpétuation du classisme, de l'homophobie, de la transphobie, de la suprématie blanche et du colonialisme; ce processus de réflexion doit en outre tenir compte du rôle de longue date des établissements de santé comme instruments de ces formes d'oppression. Cette réforme structurelle demande une vérification des programmes actuels, en partenariat avec des organisations communautaires, afin d'en revoir le contenu désuet. Les connaissances médicales liées aux enjeux 2SLGBTQIA+ doivent être incorporées à toutes les disciplines de la médecine, et les programmes d'études actuels doivent être revus de sorte qu'y

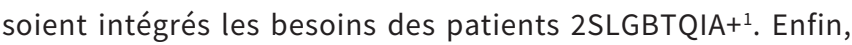
toute "norme » nationale doit veiller à l'adéquation entre le matériel de formation et les besoins des personnes 2SLGBTQIA+ de chaque région. Une analyse contextuelle de chaque établissement de santé doit être menée en vue de cerner les besoins de formation et les obstacles à l'accessibilité pour les patients et les étudiants 2SLGBTQIA+.

\section{Conclusion}

En ce moment, il n'existe au Canada aucun ensemble de compétences normalisé en matière de soins aux patients 2SLGBTQIA+. Bien qu'on puisse constater des progrès dans les établissements canadiens, aucun guide ni aucune ressource n'oriente la transformation des programmes d'études dans une optique d'amélioration des soins aux personnes 2SLGBTQIA+ au pays. Nous demandons donc à l'Association des facultés de médecine du Canada, au Collège royal des médecins et chirurgiens du Canada et au Collège des médecins de famille du Canada d'établir des normes basées sur les compétences et liées à l'obtention du permis d'exercice qui tiendront les programmes responsables d'offrir cette formation favorisant des soins de santé mieux adaptés aux besoins des personnes 2SLGBTQIA+ qui vivent au Canada.

\section{Références}

1. Integrating LGBT and DSD content into medical school curricula [AAMC videos and resources]. Washington (D.C): Association of American Medical Colleges; 2015. Accessible ici : www.aamc.org/what-we-do/diversity-inclusion/lgbt -health-resources/videos/curricula-integration (consulté le 20 juin 2020).

2. The health of LGBTQIA2 communities in Canada: report of the Standing Committee on Health. Ottawa: House of Commons; 42nd Parliament, 1st sess. June 2019. Accessible ici : www.ourcommons.ca/Content/Committee/421/HESA/ Reports/RP10574595/hesarp28/hesarp28-e.pdf (consulté le 25 juin 2020).

3. Tjepkema M. Health care use among gay, lesbian and bisexual Canadians. Health Rep 2008;19:53-64.

4. King SD, Richardson VE. Mental health for older LGBT adults. Annu Rev Gerontol Geriatr 2017;37:59-75.

5. Diagnostic and Statistical Manual of Mental Disorders (DSM-5). 5th ed. Washington (DC): American Psychiatric Association; 2013

6. Review of the public health service's response to AIDS. OTA-TM-H-24. Washington (DC): U.S. Congress Office of Technology Assessment; 1985.

7. Arbess G. Journal of the AIDS years. CMAJ 2004;171:907.

8. Rayside DM, Lindquist EA. AIDS activism and the state in Canada. Stud Polit Econ 1992;39:37-76.

9. Josephson G, Wright A. Ottawa GLBT Wellness Project: Literature review and survey instruments. Accessible ici : www.homelesshub.ca/sites/default/files/attachments /Literature_Review_and_Survey_Instruments.pdf (consulté le 25 oct. 2020).

10. Hollibaugh A, Weiss M. Queer precarity and the myth of gay affluence. New Labor Forum 2015;24:18-27. 
11. Kia H, Robinson M, MacKay J, et al. Poverty in lesbian, gay, bisexual, transgender, queer, and two-spirit (LGBTQ2S+) populations in Canada: an intersectional review of the literature. J Poverty Soc Justice 2020;28:21-54.

12. Wilkerson JM, Rybicki S, Barber CA, et al. Creating a culturally competent clinical environment for LGBT patients. J Gay Lesbian Soc Serv 2011;23:376-94.

13. Lee R. Health care problems of lesbian, gay, bisexual, and transgender patients. West J Med 2000;172:403-8.

14. Curmi C, Peters K, Salamonson Y. Lesbians' attitudes and practices of cervical cancer screening: a qualitative study. BMC Womens Health 2014;14:153.

15. Rowe D, Ng YC, O'Keefe L, et al. Providers' attitudes and knowledge of lesbian, gay, bisexual, and transgender health. Fed Pract 2017;34:28-34.

16. Mulé NJ, Ross LE, Deeprose B, et al. Promoting LGBT health and wellbeing through inclusive policy development. Int J Equity Health 2009;8:18.

17. Giblon R, Bauer GR. Health care availability, quality, and unmet need: a comparison of transgender and cisgender residents of Ontario, Canada. BMC Health Serv Res 2017;17:283.

18. Crenshaw K. Mapping the margins: intersectionality, identity politics, and violence against women of color. Stanford Law Rev 1991;43:1241-99.

19. Depelteau J, Giroux D. LGBTQ issues as Indigenous politics: Two-Spirit mobilization in Canada. In: Tremblay M, editor. Queer mobilizations: Social movement activism and Canadian public policy. Vancouver: UBC Press; 2015:64-84.

20. Frazer MS, Pruden H. Reclaiming our voices: two-spirit health and human service needs in New York State. Albany (NY): NYS DOH AIDS Institute; 2010.

21. Ristock J, Zoccole A, Passante L, et al. Aboriginal two-spirit and LGBTQ migration, mobility and health research project: Winnipeg final report, November 2010. Toronto: 2 Spirited People of the 1st Nations; 2010. Accessible ici : www.2spirits.com/PDFolder/MMHReport.pdf (consulté le 25 oct. 2020).

22. George C, Adam BA, Read SE, et al. The MaBwana Black men's study: community and belonging in the lives of African, Caribbean and other Black gay men in Toronto. Cult Health Sex 2012;14:549-62.

23. Logie $\mathrm{CH}$, Lacombe-Duncan A, Lee-Foon $\mathrm{N}$, et al. "It's for us - newcomers, LGBTQ persons, and HIV-positive persons. You feel free to be": A qualitative study exploring social support group participation among African and Caribbean lesbian, gay, bisexual and transgender newcomers and refugees in Toronto, Canada. BMC Int Health Hum Rights 2016;16:18.

24. Obedin-Maliver J, Goldsmith ES, Stewart L, et al. Lesbian, gay, bisexual, and transgender-related content in undergraduate medical education. JAMA 2011;306:971-7.

25. Wallick MM, Cambre KM, Townsend MH. How the topic of homosexuality is taught at U.S. medical schools. Acad Med 1992;67:601-3.

26. Chan B, Skocylas R, Safer JD. Gaps in transgender medicine content identified among Canadian medical school curricula. Transgend Health 2016;1:142-50.

27. Streed CG Jr, Hedian HF, Bertram A, et al. Assessment of internal medicine resident preparedness to care for lesbian, gay, bisexual, transgender, and queer/questioning patients. J Gen Intern Med 2019;34:893-8.

28. Moll J, Krieger P, Moreno-Walton L, et al. The prevalence of lesbian, gay, bisexual, and transgender health education and training in emergency medicine residency programs: What do we know? Acad Emerg Med 2014;21:608-11.

29. Irwig MS. Transgender care by endocrinologists in the United States. Endocr Pract 2016;22:832-6.

30. Nama N, Macpherson P, Sampson M, et al. Medical students' perception of lesbian, gay, bisexual, and transgender (LGBT) discrimination in their learning environment and their self-reported comfort level for caring for LGBT patients: a survey study. Med Educ Online 2017;22:1368850.

31. Turbes $\mathrm{S}, \mathrm{Krebs} \mathrm{E}$, Axtell $\mathrm{S}$. The hidden curriculum in multicultural medical education: the role of case examples. Acad Med 2002;77:209-16.

32. Eckstrand KL, Leibowitz S, Potter J, et al., editors. Chapter 3: Professional competencies to improve health care for people who are or may be LGBT, gender nonconforming, and/or born with DSD. In: Implementing curricular and institutional climate changes to improve health care for individuals who are LGBT, gender nonconforming, or born with DSD: A resource for medical educators. Washington (D.C.): Association of American Medical Colleges; 2014.
33. Dudar KJ, Ghaderi G, Gallant J, et al. Queering the medical curriculum: how to design, develop, deliver and assess learning outcomes relevant to LGBT health for health care professionals. MedEdPublish 2018 Feb. 27. doi: 10.15694/mep .2018.0000048.1.

34. Sawning S, Steinbock S, Croley R, et al. A first step in addressing medical education curriculum gaps in lesbian-, gay-, bisexual-, and transgender-related content: the University of Louisville Lesbian, Gay, Bisexual, and Transgender Health Certificate Program. Educ Health (Abingdon) 2017;30:108-14.

35. Whitehead C, Kuper A, Webster F. The conceit of curriculum. Med Educ 2012; 46:534-6.

36. Institute of Medicine (US) Committee on Lesbian, Gay, Bisexual, and Transgender Health Issues and Research Gaps and Opportunities. The health of lesbian, gay, bisexual, and transgender people: building a foundation for better understanding. Washington (D.C.): National Academies Press (US); 2011.

37. Kelley L, Chou CL, Dibble SL, et al. A critical intervention in lesbian, gay, bisexual, and transgender health: knowledge and attitude outcomes among secondyear medical students. Teach Learn Med 2008;20:248-53.

38. Frank JR, Snell LS, Cate OT, et al. Competency-based medical education: theory to practice. Med Teach 2010;32:638-45.

39. DeVita T, Bishop C, Plankey M. Queering medical education: systematically assessing LGBTQI health competency and implementing reform. Med Educ Online 2018;23:1510703.

40. Logie CH, James L, Tharao W. HIV, gender, race, sexual orientation, and sex work: a qualitative study of intersectional stigma experienced by HIV-positive women in Ontario, Canada. PLoS Med 2011;8:e1001124.

41. Mackinnon KR, Kia H, Rai N, et al. Integrating trans health knowledge through instructional design: preparing learners for a continent - not an island - of primary care with trans people. Educ Prim Care 2021 Feb. 11 [cyberpublication avant impression]. doi: 10.1080/14739879.2021.1882885.

Intérêts concurrents : Aucun déclaré.

Cet article a été révisé par des pairs.

Affiliations : Université de Toronto (Schreiber); Division d'endocrinologie et de métabolisme (Ahmad), Division d'hématologie (Scott, Imrie), Faculté de médecine, Centre de cancérologie Odette et Centre des sciences de la santé Sunnybrook (Imrie), Université de Toronto, Ont.; Département de pédiatrie et Institut d'éducation en sciences de la santé (Razack), Université McGill, Montréal, Qc.

Collaborateurs : Miranda Schreiber et Tehmina Ahmad ont contribué à la conception du travail et ont rédigé le manuscrit. Tous les auteurs ont révisé de façon critique le contenu intellectuel important du manuscrit; ils ont donné leur approbation finale pour la version destinée à être publiée et assument l'entière responsabilité de tous les aspects du travail.

Propriété intellectuelle du contenu : Il s'agit d'un article en libre accès distribué conformément aux modalités de la licence Creative Commons Attributions (CC BY-NC-ND 4.0), qui permet l'utilisation, la diffusion et la reproduction dans tout médium à la condition que la publication originale soit adéquatement citée, que l'utilisation se fasse à des fins non commerciales (c.-à-d. recherche ou éducation) et qu'aucune modification ni adaptation n'y soit apportée. Voir : https://creativecommons.org/licenses/by-nc-nd/4.0/deed.fr.

Correspondance : Tehmina Ahmad, tehmina.ahmad@mail.utoronto.ca 